\title{
Blinded by the Light: Exploiting the Deficiencies of a Laser Rangefinder for Rover Attitude Estimation
}

\author{
Jonathan D. Gammell, Chi Hay Tong, Timothy D. Barfoot \\ Institute for Aerospace Studies \\ University of Toronto \\ Toronto, Ontario, Canada \\ \{jon.gammell, chihay.tong, tim.barfoot $\} @$ utoronto.ca
}

\begin{abstract}
This paper presents a method to exploit inherent deficiencies in the sensing technology of a SICK laser rangefinder to detect sun positions from 3D lidar scans. Given the common use of SICK lidars on mobile robots, this method enables sun sensing for some existing configurations without requiring additional hardware or configuration costs. Adding sun sensing to mobile rovers has clear advantages; for example, sun vectors can be combined with an inclinometer to calculate rover orientation in an absolute reference frame and used to improve pose estimates.

The proposed sun sensing technique was verified using a SICK LMS-511 lidar mounted on a Schunk panning unit through two separate experiments. In the first experiment, the outputs of both our algorithm and a Sinclair Interplanetary SS411 digital sun sensor were compared to solar ephemeris data over an entire day. While the SS-411 has higher accuracy, the experiment showed that our lidar-based method has acceptable accuracy and a larger field of view (FOV) that covers the entire sky. In the second experiment, our sun sensing algorithm was used with an inclinometer to calculate the absolute orientation of the rover periodically during a traverse. This information was used with wheel odometry to estimate rover poses over the entire traverse, yielding more accurate results than wheel odometry alone. When including lidar-based sun measurements, the average estimate error over the entire traverse was only 8.4 metres, an $88 \%$ improvement over wheel odometry (70.4 metres). The resulting final position estimate error was 22.8 metres, or $2.76 \%$ of total distance travelled.
\end{abstract}

Keywords-lidar; sun sensing; robotics; attitude estimation; pose estimation; rover odometry

\section{INTRODUCTION}

Relative wheel odometry is the cheapest and simplest method available for estimating mobile robot position; however, without some form of external correction the estimate error grows unbounded with distance travelled. To limit error growth, odometry measurements are commonly supplemented with periodic absolute corrections. Common methods include using prior knowledge about the environment (e.g., localization against a map) or existing infrastructure (e.g., global positioning system (GPS)). It has been shown that the more accurate visual odometry (VO) techniques also show significant improvements with periodic absolutemeasurement corrections [1].

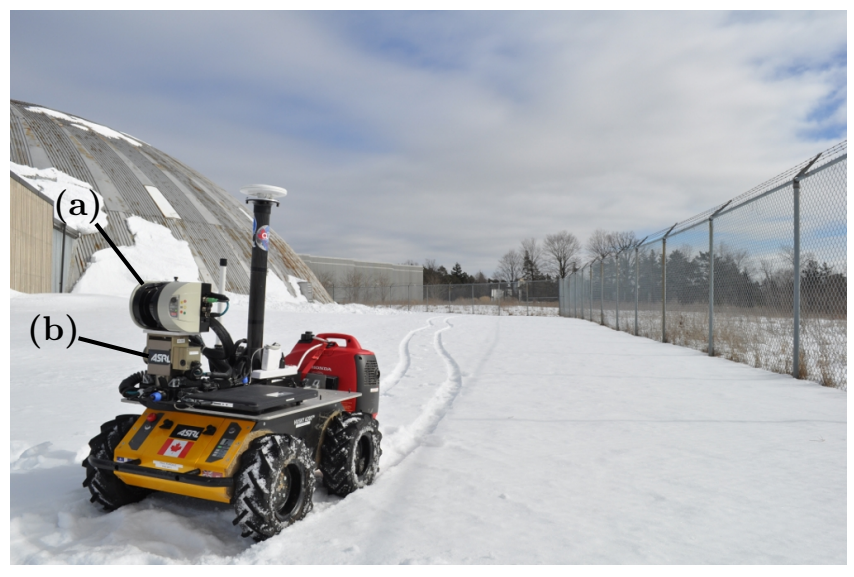

Figure 1. The ASRL Clearpath Husky A200 configured with a SICK LMS-511 scanning lidar (a) on a Schunk panning unit (b) and a Honeywell inclinometer (not shown) during the rover odometry experiment analyzed in Section IV.

Sources of localization correction are limited when deploying systems to unknown or GPS-denied environments. One common technique in planetary exploration is the use of celestial observations to calculate rover orientation or position in a global frame. Volpe [2] demonstrated such a system on the Rocky 7 rover during development for the Mars Exploration Rover (MER) program. Using a mastmounted sun sensor to measure the azimuth and elevation of the Sun and an inclinometer to measure gravity, the algorithm corrected wheel odometry measurements to achieve an average error of $6 \%$ as a function of distance over a 1 kilometre traverse. Development of this technique continued in the MER program [3], with a final design that used the onboard panoramic cameras to image the Sun [4]. Lambert et al. [1] similarly used a sun sensor and inclinometer in the VO pipeline, proposing methods to both improve the estimate accuracy and to decrease the computational burden. Gammell et al. [5] have demonstrated that similar techniques can be applied to star trackers to correct wheel odometry at night.

This paper presents a method to calculate sun vectors using 3D scanning lidars commonly found on rover plat- 


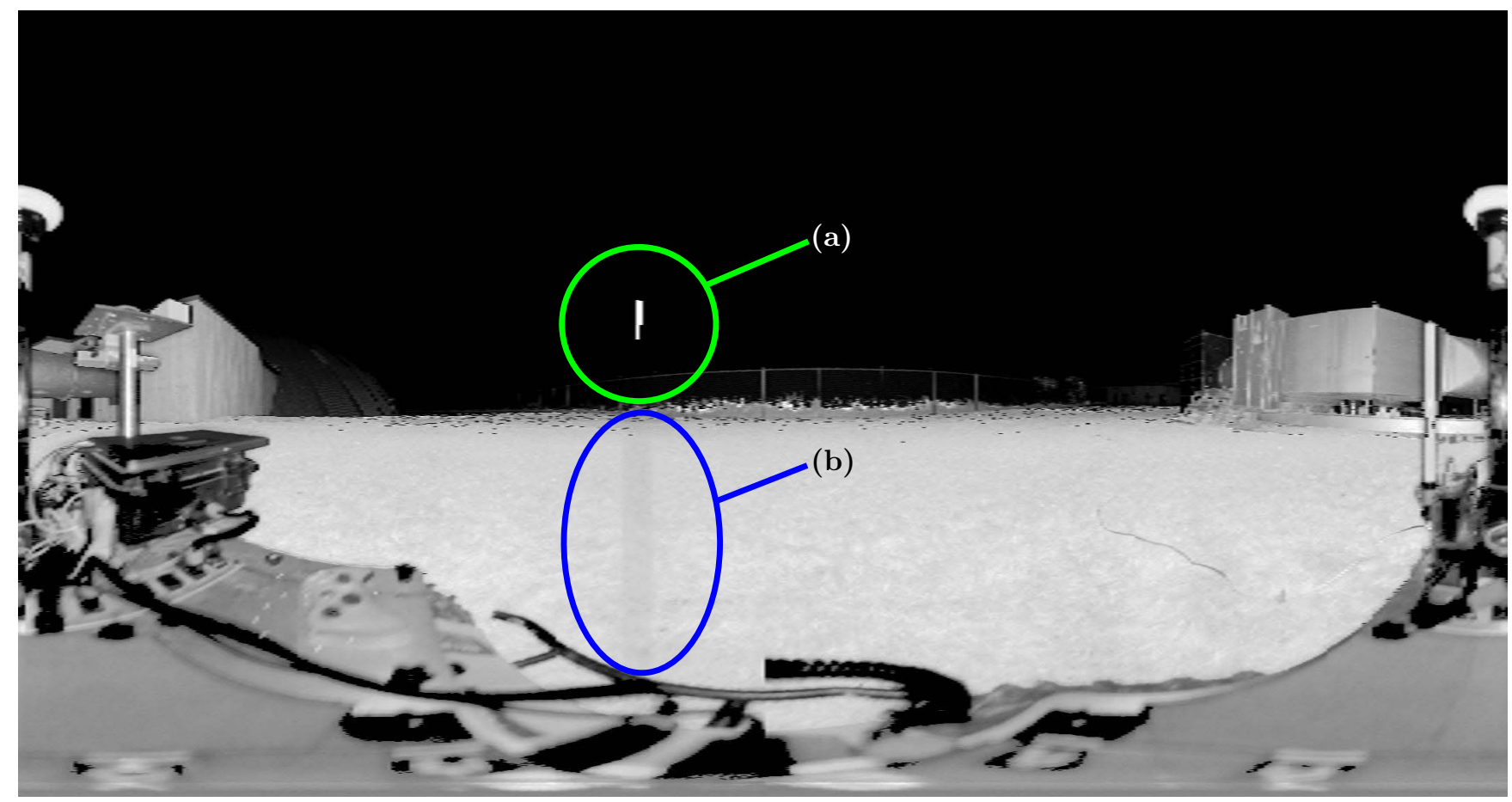

Figure 2. Reflectivity image showing a sun blob (a, green) and the extended inconsistent intensity measurements (b, blue). Dimensions are $360^{\circ} \times 190^{\circ}$.

forms and use them to improve pose estimates. The idea of using existing imaging sensors as sun detectors is not new; for example, the MERs articulated their panoramic cameras to search for and locate the Sun, using the resulting sun positions to update rover heading and point their communication antenna [4]. Presently, 3D scanning lidars are used in existing systems as sensors for obstacle detection or mapping [6], [7], as well as for lighting-invariant VO [8][10]. The proposed lidar-based technique would allow them to also operate as sun sensors in some popular configurations [11] without interfering with existing sensing operations or creating significant computational overhead.

Integrating sun sensor measurements into a system without introducing additional error or bias requires careful calibration between the sensor and the odometric frame. The work by Volpe [2] demonstrated the importance of calibration as well as the difficulty in assuring that it is maintained in real systems. If a sensor could provide both relative odometric measurements and periodic absolute corrections, it would remove a significant source of error. This paper suggests that, if coupled with lidar-based VO and an inclinometer, this could be accomplished with a 3D lidar. The result would be a system that is capable of providing both relative odometric estimates and absolute orientation corrections on existing platforms with minimal intersensor calibration.

This paper also suggests that this work could extend to the problem of large-scale, outdoor simultaneous localization and mapping (SLAM). It has been shown that SLAM results can be improved with occasional absolute measurements (e.g., GPS) [12]. The proposed sensing method would allow a single 3D lidar to provide both dense point clouds for mapping as well as strong prior estimates on orientation to aid in their alignment.

The remainder of this paper is structured as follows. The paper begins by discussing the effect of the Sun on the SICK LMS-511 and presenting our simple method to detect the Sun (Section II). This is followed by an experimental validation that compares the method's performance to both solar ephemeris data and a Sinclair Interplanetary SS-411 digital sun sensor (Section III). The results show that while the lidar method is slightly less accurate than the SS-411 at detecting the azimuth and elevation of the Sun and has a lower sampling rate, it does have a larger field of view (FOV) the covers the entire sky. The paper also presents example results of using the lidar-detected sun vectors to correct wheel odometry estimates (Section IV), and finally, some thoughts on future work to extend this novel sensing technique (Section V).

\section{LIDAR-BASED SUN DETECTION}

Lidars (LIght Detection And Ranging) are active sensors that detect the location of objects by emitting light at a known frequency and measuring the return-trip time of flight. Scanning models use mirrors or multiple light sources to image an entire scene, giving positions in a spherical 


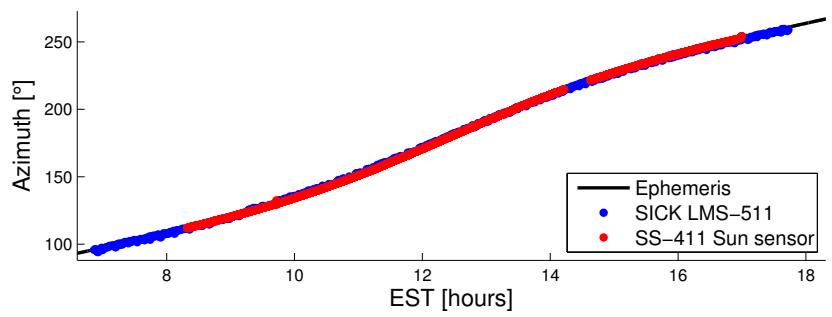

Figure 3. The azimuth of the Sun versus time calculated by the SICK LMS-511 lidar (blue), the Sinclair Interplanetary SS-411 digital sun sensor (red) and solar ephemeris data (black). Note that the SICK has a larger field of view. The lack of SS-411 data from 14:13-14:37 EST is the result of a rover shadow that occludes the sensor at that orientation.

coordinate system centred at the sensor. Most lidars also measure the intensity of the returned light, which can be used to infer the reflectivity of a measured object.

SICK lidars are popular 2D scanning lidars in robotics. These experiments used a SICK LMS-511 lidar mounted on a panning unit (the $S I C K$ ). The lidar has a range of up to 80 metres and uses a spinning mirror to scan a 905 nanometer laser in one direction over a $190^{\circ}$ linear FOV at $1 / 6^{\circ}$ spacing. It measures the range, the detection angle, and the returning light intensity. By mounting the sensor on a Schunk panning unit, the SICK can take 3D scans by panning up to $360^{\circ}$, giving azimuth, elevation, range and intensity to every measured point (Figure 1).

This configuration has been successfully used for 3D worksite mapping in planetary analogue missions [13], [14]. In the process of these experiments, it was discovered that the Sun appeared as a high-intensity, zero-range artifact in outdoor scans. Further investigations showed that the Sun emits enough light at the 905 nanometer wavelength to saturate the detector of the SICK as it passes over the Sun. This measures as an instantaneous return time, (i.e., zero range) and a maximum intensity. As the detector has a nonzero recovery time, the zero range, high intensity readings continue for a short number of measurements after passing over the Sun, resulting in a sun blob. This can be visualized by plotting the data as a reflectivity image (Figure 2). It is interesting to note that even after the sensor desaturates, the reflectivity data remain affected by their exposure to the Sun. The sensor underestimates intensity compared to similar objects, as illustrated by the dark bands below the sun blob in the reflectivity image.

Given a 3D lidar scan, we can calculate the position of the Sun by searching for these clusters of zero-range, highintensity measurements. The sun blob can be converted to a sun vector or to azimuth and elevation using knowledge of the hardware configuration. In our configuration (as the SICK scans from top down and remains saturated after moving past the Sun) the Sun is approximately located at the top of the sun blob in elevation, and in the middle in azimuth.

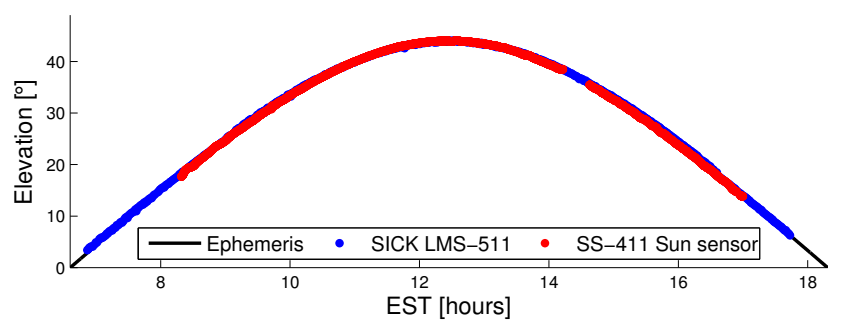

Figure 4. The elevation of the Sun versus time calculated by the SICK LMS-511 lidar (blue), the Sinclair Interplanetary SS-411 digital sun sensor (red) and solar ephemeris data (black). Note that the SICK has a larger field of view. The lack of SS-411 data from 14:13-14:37 EST is the result of a rover shadow that occludes the sensor at that orientation.

\section{Sun-SENSING PERFORMANCE}

To validate the accuracy of the proposed technique, sun vectors from 3D lidar scans acquired by the SICK were compared to simultaneously captured data from a Sinclair Interplanetary SS-411 digital sun sensor (the SS-411) and solar ephemeris data (Figures 3,4). The data were collected at the University of Toronto Institute for Aerospace Studies (UTIAS) campus in Toronto, Canada $\left(43^{\circ} 46^{\prime} 55.1^{\prime \prime} \mathrm{N}\right.$, $79^{\circ} 27^{\prime} 55.9^{\prime \prime} \mathrm{W}$ ) on March 14, 2013, a clear, cloudless day. A stationary rover equipped with the SICK and a SS-411 was used to track the Sun's traverse through the sky, starting when it first rose at 06:40 Eastern Standard Time (EST) and continuing until the Sun was hidden by nearby buildings at 17:50 EST. The SICK performed a full $360^{\circ}$ scan every 60 seconds, while the SS-411 recorded the sun position at a frequency of $1 \mathrm{~Hz}$ for the entire duration, except for a period from 14:13-14:37 EST when the SS-411 was in the shadow of the rover-mounted GPS antenna. To compare the different sensing methods to the solar ephemeris data, the rotations from the sensor frames to the ephemeris frame were estimated from a calibration dataset. This allowed the measured sun vectors to be rotated into the ephemeris frame and then converted to azimuth and elevation.

\section{A. Solar Ephemeris Calibration}

Each individual sensor measures the position of the Sun in its local frame. The rotation between a sensor frame and the ephemeris frame, $\mathbf{C}_{E S}$, can be calculated from a dataset consisting of $M$ unit sun vectors from the sensor, $\mathbf{u}_{S_{i}}$, and the solar ephemeris data, $\mathbf{u}_{E_{i}}$. The best estimate of the rotation from the sensor to ephemeris frame, $\mathbf{C}_{E S}^{*}$, can be written as a classic Wahba problem [15] determining the rotation matrix that minimizes a cost function,

$$
\begin{aligned}
\mathbf{C}_{E S}^{*} & :=\underset{\mathbf{C}_{E S}}{\arg \min }\left\{J\left(\mathbf{C}_{E S}\right)\right\}, \\
J\left(\mathbf{C}_{E S}\right) & :=\frac{1}{2} \sum_{i=1}^{M}\left\|\mathbf{u}_{E_{i}}-\mathbf{C}_{E S} \mathbf{u}_{S_{i}}\right\|^{2} .
\end{aligned}
$$




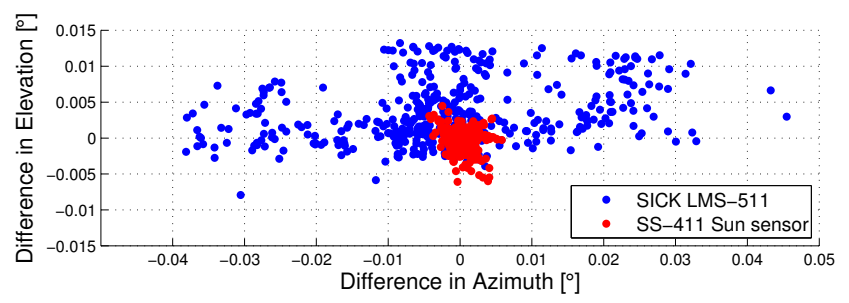

Figure 5. Measurement error, in degrees, of the SICK-based sun sensor (blue) and a SS-411 digital sun sensor (red) as calculated from solar ephemeris data.

For our experiment, a singular value decomposition (SVD) method [16], [17] was used to calculate the least-squared solution to (1). The calibration vectors are columnated into matrices, and multiplied together,

$$
\mathbf{W}:=\left[\begin{array}{llll}
\mathbf{u}_{E_{1}} & \mathbf{u}_{E_{2}} & \ldots & \mathbf{u}_{E_{M}}
\end{array}\right]\left[\begin{array}{llll}
\mathbf{u}_{S_{1}} & \mathbf{u}_{S_{2}} & \ldots & \mathbf{u}_{S_{M}}
\end{array}\right]^{T} .
$$

The result is then factored,

$$
\mathbf{U S V}^{T}:=\mathbf{W}
$$

where $\mathbf{U}$ and $\mathbf{V}$ are unitary matrices and $\mathbf{S}$ is rectangular diagonal matrix, as per SVD. The optimal estimate of the rotation is then given by,

$$
\mathbf{C}_{E S}^{*}=\mathbf{U}\left[\begin{array}{ccc}
1 & 0 & 0 \\
0 & 1 & 0 \\
0 & 0 & |\mathbf{U}||\mathbf{V}|
\end{array}\right] \mathbf{V}^{T}
$$

\section{B. Results}

Over the entire day, 637 sun vectors were calculated by the SICK from 660 scans. When evaluating the SICK and SS-411 to the solar ephemeris data, we compared only data occurring in the FOVs of both sensors. While the SICK can detect the Sun over the entire sky, the SS-411 has a $\pm 70^{\circ}$ FOV centred about the vertical axis. There are 483 SICK measurements with a corresponding SS-411 measurement within 1 second.

This set of paired measurements was split into a calibration set of 50 measurements used to estimate the rotation between each sensor and the solar ephemeris data, and a test set of 433 measurements used to evaluate the performance of the sensors (Table I, Figure 5). The results show that the SICK has less variance in elevation than in azimuth with bias in both measurements and that the SS-411 is more accurate.

The elevation performance of the SICK depends on the method used to convert the sun blob into a sun vector and the scanning resolution of the lidar. Better performance may be realized from more advanced sun blob processing techniques and higher scan resolution. The azimuth performance of the SICK depends on the synchronization between the lidar and the panning unit. Since the pan angle of a lidar measurement is calculated from the lidar and the panning unit timestamps, improving the synchronization between these two clocks could improve performance [18].
Table I

MEASUREMENT ERROR, IN DEGREES, OF THE SICK-BASED SUN SENSOR AND A SS-411 DIGITAL SUN SENSOR AS CALCULATED FROM SOLAR EPHEMERIS DATA.

\begin{tabular}{ccccc}
\hline & \multicolumn{2}{c}{ Azimuth } & \multicolumn{2}{c}{ Elevation } \\
\cline { 2 - 5 } & Mean & St. dev. & Mean & St. dev. \\
\hline SS-411 & 0.031 & 0.086 & -0.011 & 0.081 \\
SICK & -0.106 & 0.875 & 0.199 & 0.243 \\
\hline
\end{tabular}

\section{ODOMETRY CORRECTIONS}

To demonstrate the value of the lidar-based sun sensing technique, the Clearpath Husky A200 was equipped with the SICK and an inclinometer and driven 826 metres through the UTIAS campus while acquiring $15,360^{\circ}$ lidar scans (Figure 6). At each scan location, the sun vector was calculated from the 3D lidar scan and combined with inclinometer measurements and solar ephemeris data to estimate the absolute orientation of the rover. These periodic orientations were used as corrections to the pose-estimation problem using a batch estimator.

\section{A. Batch Optimization}

The absolute orientation measurements from the Sun were used to improve the relative motion from the wheel odometry in a batch optimization formulation [5]. Expressing the relative odometric measurements as 6 degree-of-freedom (DOF) transformation matrices, $\tilde{\mathbf{T}}_{k, k-1}$, with associated covariances, $\mathbf{U}_{k, k-1}$, and the absolute orientation measurements as 3 DOF rotation matrices, $\tilde{\mathbf{C}}_{k, 0}$, with associated covariances, $\mathbf{P}_{k, 0}$, we wish to find the set of optimal estimates whose resulting measurement errors, $\delta \boldsymbol{\epsilon}_{k, k-1}$ and $\delta \boldsymbol{\psi}_{k, 0}$, minimize a cost function,

$$
J:=\frac{1}{2} \sum_{k}\left(\delta \boldsymbol{\epsilon}_{k, k-1}^{T} \mathbf{U}_{k, k-1}^{-1} \delta \boldsymbol{\epsilon}_{k, k-1}+\delta \boldsymbol{\psi}_{k, 0}^{T} \mathbf{P}_{k, 0}^{-1} \delta \boldsymbol{\psi}_{k, 0}\right)
$$

The measurement errors are defined in terms of the estimate, $\mathbf{T}_{k, 0}$, as

$$
\begin{aligned}
e^{-\delta \boldsymbol{\epsilon}_{k, k-1}^{\boxplus}} & :=\tilde{\mathbf{T}}_{k, k-1} \mathbf{T}_{k-1,0} \mathbf{T}_{k, 0}^{-1} \\
e^{-\delta \boldsymbol{\psi}_{k, 0}^{\times}} & :=\tilde{\mathbf{C}}_{k, 0} \mathbf{C}_{k, 0}^{T},
\end{aligned}
$$

where $\mathbf{T}_{k, 0}$ is a $4 \times 4$ transformation matrix consisting of a rotation from 0 to $k, \mathbf{C}_{k, 0}$, and translation from 0 to $k$ expressed in frame $0, \mathbf{r}_{0}^{k 0}$,

$$
\mathbf{T}_{k, 0}:=\left[\begin{array}{cc}
\mathbf{C}_{k, 0} & -\mathbf{C}_{k, 0} \mathbf{r}_{0}^{k 0} \\
\mathbf{0}^{T} & 1
\end{array}\right]
$$

The operator, $(\cdot)^{\boxplus}$, is defined for a $6 \times 1$ vector, $\mathbf{w}$, as

$$
\mathbf{w}^{\boxplus}=\left[\begin{array}{l}
\mathbf{u} \\
\mathbf{v}
\end{array}\right]^{\boxplus}:=\left[\begin{array}{cc}
\mathbf{v}^{\times} & -\mathbf{u} \\
\mathbf{0}^{T} & 0
\end{array}\right],
$$




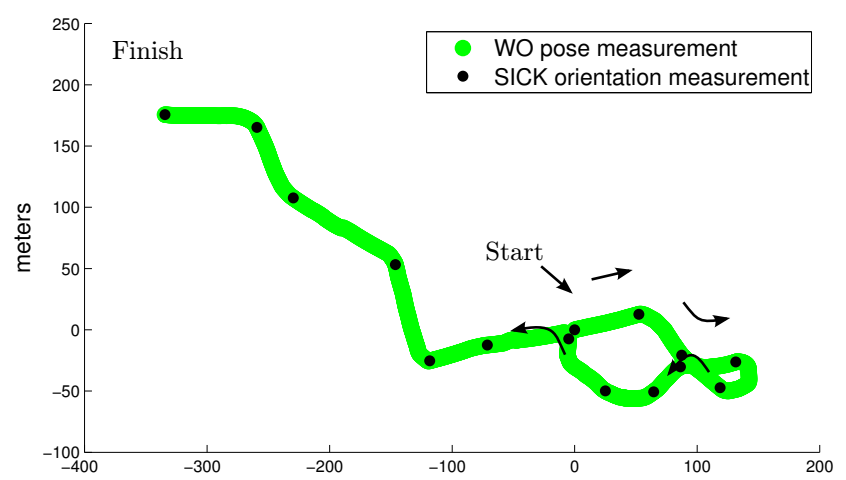

Figure 6. The odometry dataset collected at UTIAS consisting of wheel odometry pose estimates (green) and sun vectors from lidar scans (black). Arrows serve to illustrate the direction of the traverse.

and the operator, $(\cdot)^{\times}$, is defined for a $3 \times 1$ vector, $\mathbf{u}$, as

$$
\mathbf{u}^{\times}=\left[\begin{array}{l}
u_{1} \\
u_{2} \\
u_{3}
\end{array}\right]^{\times}:=\left[\begin{array}{ccc}
0 & -u_{3} & u_{2} \\
u_{3} & 0 & -u_{1} \\
-u_{2} & u_{1} & 0
\end{array}\right] .
$$

The minimum of (2) can be found iteratively with GaussNewton. We approximate the current measurement errors as a function of the previous iteration's errors, $\delta \overline{\boldsymbol{\epsilon}}_{k, k-1}$ and $\delta \overline{\boldsymbol{\psi}}_{k, 0}$, as

$$
\begin{aligned}
\delta \boldsymbol{\epsilon}_{k, k-1} & \approx \delta \overline{\boldsymbol{\epsilon}}_{k, k-1}-\mathbf{H}_{k, k-1} \mathbf{B}_{k, k-1} \delta \mathbf{x}, \\
\delta \boldsymbol{\psi}_{k, 0} & \approx \delta \overline{\boldsymbol{\psi}}_{k, 0}-\mathbf{G B}_{k, 0} \delta \mathbf{x},
\end{aligned}
$$

with

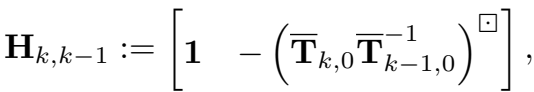

$$
\begin{aligned}
& \mathbf{G}:=\left[\begin{array}{llll}
\mathbf{0} & \mathbf{1} & \mathbf{0} & \mathbf{0}
\end{array}\right], \\
& \mathbf{B}_{k, k-1} \delta \mathbf{x}:=\left[\begin{array}{c}
\delta \boldsymbol{\pi}_{k, 0} \\
\delta \boldsymbol{\pi}_{k-1,0}
\end{array}\right] \text {. }
\end{aligned}
$$

Where $\mathbf{1}$ as the identity matrix, and the operator, $(\cdot)^{\square}$, is defined for any transformation matrix, $\mathbf{T}$, as

$$
\mathbf{T}^{\square}=\left[\begin{array}{cc}
\mathbf{C} & -\mathbf{C r} \\
\mathbf{0}^{T} & 1
\end{array}\right]^{\square}:=\left[\begin{array}{cc}
\mathbf{C} & \mathbf{C r} \times \\
\mathbf{0} & \mathbf{C}
\end{array}\right] .
$$

The projection matrix, $\mathbf{B}_{k . k-1}$, selects the perturbations, $\delta \boldsymbol{\pi}_{k, 0}$ and $\delta \boldsymbol{\pi}_{k-1,0}$, for the $k$ and $k-1$ poses from the entire state, $\delta \mathbf{x}$. New estimates can then be expressed in terms of perturbations on the previous estimates, $\overline{\mathbf{T}}_{k, 0}$, as

$$
\mathbf{T}_{k, 0}=e^{-\delta \boldsymbol{\pi}_{k, 0}^{\boxplus}} \overline{\mathbf{T}}_{k, 0} .
$$

The process is repeated until a suitable convergence criterion is reached.

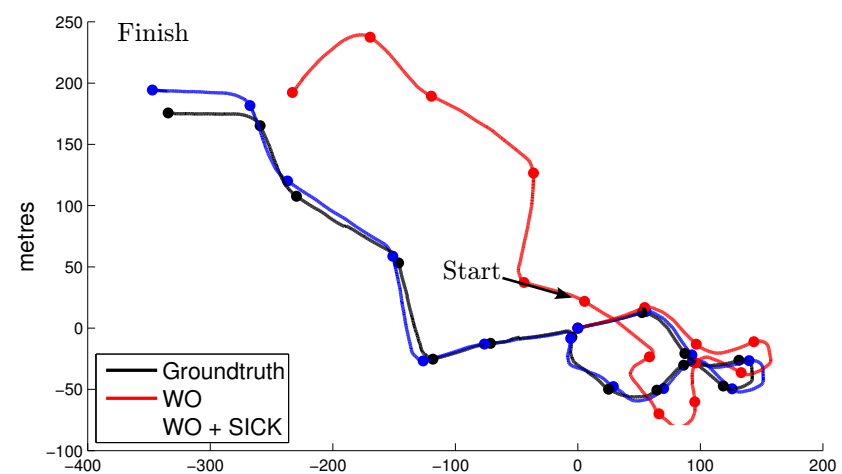

Figure 7. Estimates produced from wheel odometry corrected with lidarbased sun vectors (blue) plotted with the DGPS groundtruth (black), and the wheel odometry (red). Dots indicate the locations at which 3D lidar scans were acquired to measure absolute orientation from the Sun.

\section{B. Results}

The batch estimator with lidar-based sun measurements was compared to groundtruth calculated from post-processed differential GPS gathered by an onboard receiver. The first 100 metres of the traverse were used to align the groundtruth to the local rover frame. As expected, the sun-vectorcorrected method provided a more accurate estimate than wheel odometry alone (Figure 7). The average estimate error over the traverse was 8.4 metres, an $88 \%$ improvement over the average wheel odometry error of 70.4 metres (Table II). The final position error was 22.8 metres, or $2.76 \%$ of total distance travelled.

\section{Discussion \& Future Work}

Orientation errors cause uncorrected pose estimates to grow superlinearly without bound. Providing frequent and accurate orientation corrections can limit this to linear growth [1]. Therefore, it is expected that the results presented in Section IV can be extended to any system using a 3D lidar and requiring accurate pose estimates, including VO and SLAM. Doing so would leverage hardware commonly found on existing platforms for mapping and obstacle detection into a simple, yet improved, estimation system that requires minimal intersensor calibration.

While the presented method was demonstrated and evaluated on a SICK LMS-511 lidar, the technique should be extensible to other makes and models. The presented method could be adapted with at most minor modifications; the method of extracting measurements from sun blobs may have to be modified to reflect different scanning mechanisms. The only requirements are that the lidar must use a wavelength strongly emitted by the Sun and also measure the intensity of the returning light. Other popular lidars in robotics that may potentially work include models made by Velodyne and Hokuyo. 

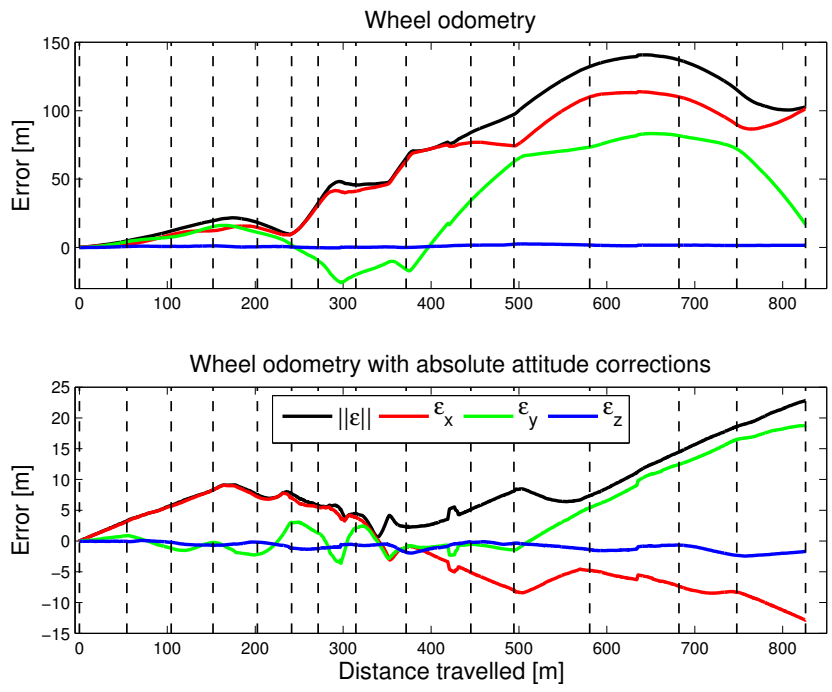

Figure 8. Position estimate error versus distance travelled showing that using lidar-based sun vectors to correct wheel odometry significantly reduces error. Dotted lines indicate the locations at which sun vectors were acquired from 3D lidar scans to provide absolute orientation corrections. Note the difference in scale between the wheel odometry and sun-vectorcorrected estimates.

With an inclinometer and solar ephemeris data, a lidar could be used to provide both relative odometry estimates through lidar-based VO [8]-[10], and absolute orientation corrections from celestial calculations. A similar configuration could also be used in SLAM problems to provide both dense point clouds for mapping as well as strong estimates on prior orientation to aid in their alignment.

\section{CONCLUSION}

This paper presents a novel method to detect the Sun using a common scanning 3D lidar. The presented technique is simple, accurate, and computationally inexpensive. The required hardware is common on many systems and the adoption of the presented technique does not interfere with existing sensing operations or require additional sensors or associated calibrations. Experiments showed the lidar method to have lower accuracy and measurement frequency than a digital sun sensor but an expanded FOV.

With only one additional sensor, an inclinometer, the technique can be expanded to provide full rover attitude estimates from solar ephemeris data. These attitude estimates can be used to improve the accuracy of pose estimates in a wide range of applications, including VO and SLAM. The paper presents an example of using sun vectors from a SICK LMS-511 mounted on a Schunk panning unit to correct wheel odometry on a dataset of over 800 metres. The resulting estimate had an average error of 8.4 metres, a $88 \%$ improvement over basic wheel odometry (70.4 metres), and a final position error of 22.8 metres, or $2.76 \%$ of total distance travelled.
Table II

ERROR IN METERS, $\boldsymbol{\epsilon}$, OF THE WHEEL ODOMETRY (WO) AND LIDAR-BASED SUN-VECTOR-CORRECTED (WO + SICK) ESTIMATES EXPRESSED IN THE LOCAL ROVER FRAMES

\begin{tabular}{ccccccc}
\hline & \multicolumn{3}{c}{ WO } & \multicolumn{3}{c}{ WO + SICK } \\
\cline { 2 - 7 } & Mean & Max. & Final & Mean & Max. & Final \\
$\|\epsilon\|$ & 70.4 & 140.9 & 102.8 & 8.4 & 22.8 & 22.8 \\
$\epsilon_{x}$ & 59.2 & 113.9 & 101.4 & -1.5 & 12.9 & -12.9 \\
$\epsilon_{y}$ & 30.9 & 83.4 & 16.7 & 3.8 & 18.8 & 18.8 \\
$\epsilon_{z}$ & 1.1 & 2.6 & 1.6 & -0.9 & 2.4 & -1.7 \\
\hline
\end{tabular}

\section{ACKNOWLEDGMENT}

The authors wish to thank Mr. Doug Sinclair from Sinclair Interplanetary for the donation of the SS-411 digital sun sensor, Prof. John Enright from Ryerson University for advice on celestial measurements, Clearpath Robotics for the Husky A200, and the Canadian Space Agency (CSA) the Defence Research and Development Canada (DRDC), the Natural Sciences and Engineering Research Council of Canada (NSERC), and MDA Space Systems for their financial and in-kind support.

\section{REFERENCES}

[1] A. Lambert, P. T. Furgale, T. D. Barfoot, and J. Enright, "Field testing of visual odometry aided by a sun sensor and inclinometer," Journal of Field Robotics, special issue on "Space Robotics", vol. 29, no. 3, pp. 426-444, May - Jun. 2012.

[2] R. Volpe, "Mars rover navigation results using sun sensor heading determination," in Proc. of the IEEE/RSJ Int. Conf. on Intelligent Robots and Systems (IROS), vol. 1, 1999, pp. 460-467.

[3] E. T. Baumgartner, H. Aghazarian, A. Trebi-Ollennu, T. L. Huntsberger, and M. S. Garrett, "State estimation and vehicle localization for the FIDO rover," Proc. of SPIE Sensor Fusion and Decentralized Control in Robotic Systems III, vol. 4196, pp. 329-336, 2000.

[4] K. Ali, C. Vanelli, J. Biesiadecki, M. Maimone, Y. Cheng, A. San Martin, and J. Alexander, "Attitude and position estimation on the Mars exploration rovers," in Proc. of the IEEE Int. Conf. on Systems, Man and Cybernetics, vol. 1, Oct. 2005, pp. $20-27$ Vol. 1.

[5] J. D. Gammell, C. H. Tong, P. Berczi, S. Anderson, T. D. Barfoot, and J. Enright, "Rover odometry aided by a star tracker," in Proc. of the IEEE Aerospace Conference, Big Sky, MT, 2-9 Mar. 2013.

[6] S. Thrun, M. Montemerlo, H. Dahlkamp, D. Stavens, A. Aron, J. Diebel, P. Fong, J. Gale, M. Halpenny, G. Hoffmann, K. Lau, C. Oakley, M. Palatucci, V. Pratt, P. Stang, S. Strohband, C. Dupont, L.-E. Jendrossek, C. Koelen, C. Markey, C. Rummel, J. van Niekerk, E. Jensen, P. Alessandrini, G. B. B. Davies, S. Ettinger, A. Kaehler, A. Nefian, and P. Mahoney, "Stanley: The robot that won the DARPA grand challenge," Journal of Field Robotics, vol. 23, no. 9, pp. 661692, 2006. 
[7] C. Urmson, J. Anhalt, D. Bagnell, C. Baker, R. Bittner, M. N. Clark, J. Dolan, D. Duggins, T. Galatali, C. Geyer, M. Gittleman, S. Harbaugh, M. Hebert, T. M. Howard, S. Kolski, A. Kelly, M. Likhachev, M. McNaughton, N. Miller, K. Peterson, B. Pilnick, R. Rajkumar, P. Rybski, B. Salesky, Y.-W. Seo, S. Singh, J. Snider, A. Stentz, W. Whittaker, Z. Wolkowicki, J. Ziglar, H. Bae, T. Brown, D. Demitrish, B. Litkouhi, J. Nickolaou, V. Sadekar, W. Zhang, J. Struble, M. Taylor, M. Darms, and D. Ferguson, "Autonomous driving in urban environments: Boss and the urban challenge," Journal of Field Robotics, vol. 25, no. 8, pp. 425-466, 2008.

[8] H. J. Dong and T. D. Barfoot, "Lighting-invariant visual odometry using lidar intensity imagery and pose interpolation," in Proc. of the Int. Conf. on Field and Service Robotics (FSR), Matsushima, Japan, 16-19 Jul. 2012.

[9] C. McManus, P. T. Furgale, B. E. Stenning, and T. D. Barfoot, "Lighting-invariant visual teach and repeat using appearancebased lidar," Journal of Field Robotics, 2013.

[10] C. Tong and T. D. Barfoot, "Gaussian Process Gauss-Newton for 3D laser-based visual odometry," in Proc. of the IEEE Conf. on Robotics and Automation (ICRA), Karlsruhe, Germany, 6-10 May 2013.

[11] A. Harrison and P. Newman, "High quality 3D laser ranging under general vehicle motion," in Proc. of the IEEE Int. Conf. on Robotics and Automation (ICRA), Pasadena, California, Apr. 2008.

[12] S. Thrun and M. Montemerlo, "The graph slam algorithm with applications to large-scale mapping of urban structures," The Int. Journal of Robotics Research, vol. 25, no. 5-6, pp. 403-429, 2006.

[13] R. S. Merali, C. Tong, J. Gammell, J. Bakambu, E. Dupuis, and T. D. Barfoot, "3D surface mapping using a semiautonomous rover: A planetary analog field experiment," in Proc. of the 2012 Int. Symposium on Artificial Intelligence, Robotics and Automation in Space (i-SAIRAS), Turin, Italy, 4-6 Sep. 2012.

[14] L.-P. Berczi, J. D. Gammell, C. H. Tong, T. D. Barfoot, and M. Daly, "Rover-based method for detecting methane from a seep," in Proc. of the 10th Canadian Conf. on Computer and Robot Vision (CRV), Regina, SK, Canada, 28-31 May 2013.

[15] G. Wahba, "A least squares estimate of satellite attitude," Society for Industrial and Applied Mathematics (SIAM) Review, vol. 7, p. 409, 1965.

[16] F. L. Markley, "Attitude determination using vector observations and the singular value decomposition," Journal of the Astronautical Sciences, vol. 36, no. 3, pp. 245-258, Jul. - Sep. 1988.

[17] S. Umeyama, "Least-squares estimation of transformation parameters between two point patterns," IEEE Transactions on Pattern Analysis and Machine Intelligence, vol. 13, no. 4, pp. 376 -380, Apr. 1991.

[18] A. Harrison and P. Newman, "TICSync: Knowing when things happened," in Proc. of the IEEE Conf. on Robotics and Automation (ICRA), Shanghai, China, May 2011. 